\title{
Comparative genome and phenotypic analysis of three Clostridioides difficile strains isolated from a single patient provide insight into multiple infection of $C$. difficile
}

Uwe Groß ${ }^{1 \dagger}$, Elzbieta Brzuszkiewicz ${ }^{2 \dagger}$, Katrin Gunka ${ }^{1}$, Jessica Starke ${ }^{2}$, Thomas Riedel $^{3,4}$, Boyke Bunk ${ }^{3}$, Cathrin Spröer ${ }^{3}$, Daniela Wetzel ${ }^{1}$, Anja Poehlein ${ }^{2}$, Cynthia Chibani ${ }^{2}$, Wolfgang Bohne ${ }^{1}$, Jörg Overmann ${ }^{3,4}$, Ortrud Zimmermann ${ }^{1}$, Rolf Daniel ${ }^{2}$ and Heiko Liesegang ${ }^{2^{*}}$ (D)

\begin{abstract}
Background: Clostridioides difficile infections (CDI) have emerged over the past decade causing symptoms that range from mild, antibiotic-associated diarrhea (AAD) to life-threatening toxic megacolon. In this study, we describe a multiple and isochronal (mixed) CDI caused by the isolates DSM 27638, DSM 27639 and DSM 27640 that already initially showed different morphotypes on solid media.

Results: The three isolates belonging to the ribotypes (RT) 012 (DSM 27639) and 027 (DSM 27638 and DSM 27640) were phenotypically characterized and high quality closed genome sequences were generated. The genomes were compared with seven reference strains including three strains of the RT 027, two of the RT 017, and one of the RT 078 as well as a multi-resistant RT 012 strain. The analysis of horizontal gene transfer events revealed gene acquisition incidents that sort the strains within the time line of the spread of their RTs within Germany. We could show as well that horizontal gene transfer between the members of different RTs occurred within this multiple infection. In addition, acquisition and exchange of virulence-related features including antibiotic resistance genes were observed. Analysis of the two genomes assigned to RT 027 revealed three single nucleotide polymorphisms (SNPs) and apparently a regional genome modification within the flagellar switch that regulates the fli operon.
\end{abstract}

Conclusion: Our findings show that (i) evolutionary events based on horizontal gene transfer occur within an ongoing $\mathrm{CDI}$ and contribute to the adaptation of the species by the introduction of new genes into the genomes, (ii) within a multiple infection of a single patient the exchange of genetic material was responsible for a much higher genome variation than the observed SNPS.

Keywords: Clostridioides difficile, Clostridium difficile, Multiple infection, Genetic switch, Genomic adaptation, Horizontal gene transfer

\footnotetext{
* Correspondence: hlieseg@gwdg.de

${ }^{\dagger}$ Equal contributors

2Department of Genomic and Applied Microbiology \& Göttingen Genomics Laboratory, Institute of Microbiology and Genetics, Georg-August-University, Göttingen, Germany

Full list of author information is available at the end of the article
} 


\section{Background}

Clostridioides difficile is a Gram-positive, obligate anaerobic spore-forming bacterium, which can act as nosocomial human pathogen colonizing the intestinal tract and causing disease [1]. Symptoms of $C$. difficile infection (CDI) can range from mild diarrhea to pseudomembranous colitis or life-threatening toxic megacolon [2, 3]. C. difficile has been described by Miller et al. in 2011 [4] together with methicillin-resistant Staphylococcus aureus (MRSA) as the most common cause of nosocomial infections in the United States. Consequently, C. difficile was prioritized in the highest rank for surveillance and epidemiological research [5].

Strains of $C$. difficile are currently distinguished by PCR ribotyping (comparison of pattern of PCR products of the 16S-23S rRNA intergenic spacer region), which allows to follow epidemic infection routes [6]. Ribotype (RT) 027 was responsible for dynamic increase of CDIs in North-America and Europe, which quadrupled the number of CDI victims between 2004 and 2007 [7]. However, this epidemic outbreak had been successfully controlled with a decrease in CDI infection in 2008 [8, 9]. The infection line and the spread of the outbreak in Germany has been traced by a genome sequence-based BEAST-analysis [10]. Thus, the mutation rate of the strains challenged by the immune system of the patients was sufficient to track the transmission of the pathogen $C$. difficile from patient to patient. The dynamic adaptation of pathogens challenged by a host immune defense is the reason why host/pathogen systems have been used as model-system to investigate the speed of genome evolution $[11,12]$. Genome analysis of representative strains of $C$. difficile from the PCR ribotypes 001, 017, 027 and 078 revealed the presence of distinct genomes [7]. The investigated genomes contain an extensive pan-genome shaped by horizontal gene transfer (HGT). The quantification of HGT events revealed that $11 \%$ of the whole genome is consisting of mobile elements [7, 13-15]. The described genome diversity of the species contrasts with the phenotypic similarity of isolated strains with respect to growth, virulence and pathogenicity [14]. Notably, the genes encoding the toxins TcdA and TcdB assigned to the CDI virulence [16, 17] are located on the pathogenicity locus (PaLoc) which constitutes a genomic island. This indicates that HGT is involved in the evolution of toxigenic $C$. difficile strains [18]. In $E$. coli, HGT events between different strains have been identified as an important source for new combination of virulence factors and thus for the emergence of novel pathotypes [19]. In CDI cases, multiple infections by $C$. difficile strains, which are different in morphology and virulence, have been observed [20,21], thereby indicating the opportunity to exchange genetic material through HGT events between the strains involved. In this study, we present a comparative phenotypic and genome analysis of three morphologically different $C$. difficile strains isolated from a single patient. Two of the strains belong to RT 027 (DSM 27638 and DSM 27640) and one to RT 012 (DSM 27639).

\section{Methods}

\section{Isolation of strains}

A stool sample from a patient with diarrhea was cultivated anaerobically on Clostridium difficile (CLO) agar plates (bioMérieux, Nürtingen, Germany), which is a selective medium for $C$. difficile. After $48 \mathrm{~h}$ of cultivation at $37{ }^{\circ} \mathrm{C}$, colonies characteristic for $C$. difficile were visible and were confirmed by MALDI-TOF mass spectrometry (Biotyper, Bruker Daltonics, Bremen, Germany) with score values of $\geq 2000$. Isolated strains were deposited at the DSMZ under the accession numbers DSM 27638, DSM 27639 and DSM 27640.

\section{Phenotypic characterization \\ General growth conditions}

C. difficile strains were grown at $37{ }^{\circ} \mathrm{C}$ in an anaerobic chamber (Coy Laboratory Products, Michigan USA) under an atmosphere of $5 \% \mathrm{CO}_{2}, 5 \% \mathrm{H}_{2}, 90 \% \mathrm{~N}_{2}$ or in a jar (Merck, Darmstadt, Germany) using an anaerobic gas pack (bioMérieux, Nürtingen, Germany). For liquid cultures, BHIS (brain heart infusion; BD, Heidelberg, Germany) supplemented with $0.5 \%$ yeast extract (BD, Heidelberg, Germany) and 0.03\% L-cysteine (Sigma, Taufkirchen, Germany) was used. Cultivation on plates was performed using chromID ${ }^{\mathrm{Tm}} \mathrm{C}$. difficile agar (CDIFF), Clostridium difficile agar (CLO) or Columbia agar with $5 \%$ sheep blood (COS) (bioMérieux Nürtingen, Germany). Plates were incubated for 24 to $48 \mathrm{~h}$.

\section{Sporulation assay}

Sporulation rates were determined according to Burns et al., [22]. Briefly, an overnight culture of the respective strain was diluted 1:100 with fresh BHIS and was grown until an optical density $\left(\mathrm{OD}_{600}\right)$ of 0.2 to 0.4 . This culture was again diluted 1:100 with fresh BHIS and cultivated for five days. An aliquot of the culture was heated for $25 \mathrm{~min}$ at $60{ }^{\circ} \mathrm{C}$ to kill the vegetative cells. Dilution series of an untreated and the heated sample with sterile saline was performed and spotted on CDIFF plates. Colony forming units (CFU) were analyzed after $24 \mathrm{~h}$ of incubation.

\section{Co-cultivation of $C$. difficile strains}

An overnight culture was adjusted to an $\mathrm{OD}_{600}$ of 0.1 in fresh BHIS. $400 \mu \mathrm{l}$ of the cells were incubated in one well of a 24-well plate (Greiner Bio-One, Frickenhausen, Germany). A ThinCert ${ }^{\mathrm{Tm}}$ insert (pore size $0.4 \mu \mathrm{m}$ ) was placed in this well and $400 \mu \mathrm{l}$ of the respective $C$. difficile strain was added. The insert allows the diffusion of 
metabolites between the two cultures but not of cells or spores. A dilution series of the cells at the time point zero was spotted on CDIFF plates to ensure that equal amounts of the respective strains were used for co-cultivation. After $24 \mathrm{~h}$ of incubation, the cells in the wells and inserts were resuspended thoroughly and adjusted to equal volumes. A dilution series of the cells was performed on CDIFF agar plates and incubated for $24 \mathrm{~h}$. To assess whether the insert membrane is tight for $C$. difficile, controls were performed with (i) bacteria in the insert and sterile medium in the well and (ii) sterile medium in the insert and bacteria in the well. After plating aliquots on CDIFF plates, no colonies were formed after a $24 \mathrm{~h}$ incubation time for the medium controls, indicating that bacteria do not pass the insert membrane in relevant numbers within the $24 \mathrm{~h}$ incubation time.

\section{Mobility assay}

The motility of $C$. difficile strains was tested by stabinoculation of a fresh single colony grown on BHI-agar in 0.175-0.3\% semi-solid BHI-agar. Anaerobic incubation at $37{ }^{\circ} \mathrm{C}$, and monitoring of the developed diffusion radius around the inoculation stab for the following days, were performed. $0.3 \%$ BHI-agar plates were prepared and anaerobically incubated for $3 \mathrm{~h}$ before inoculation. Plates were inoculated in the center of the plate with a single fresh $C$. difficile colony and incubated under anoxic condition $\left(5 \% \mathrm{H}_{2}, 5 \% \mathrm{CO}_{2}, 90 \% \mathrm{~N}_{2}\right)$ at $37^{\circ} \mathrm{C} .1$ and $2 \mathrm{~d}$ post inoculation, plates were removed from the anaerobic atmosphere for scanning of the plates. Motility assay on agar plates was performed incubating plates upside down and with the lid upturned to avoid problems with condensing water. Hungate tubes containing semi-solid $0.175 \%$ BHI-agar were incubated anaerobically overnight before inoculation. Agar was inoculated in the center of the hungate tube with a single fresh $C$. difficile colony using an inoculation needle in four replicates and incubated anaerobically $\left(5 \% \mathrm{H}_{2}, 95 \% \mathrm{~N}_{2}\right)$ at $37{ }^{\circ} \mathrm{C}$. Diffusion radius around the inoculation stab was monitored taking pictures 1, 2 and $3 \mathrm{~d}$ post inoculation.

\section{Transmission electron microscopy}

For visualization of $C$. difficile cells via Transmission Electron Microscopy (TEM) cells were negatively stained using $1 \%(w / v)$ uranyl acetate. C. difficile cultures were inoculated and grown to exponential or stationary phase. Cultures were either used directly for sample preparation, or were previously washed to get rid of media ingredients. Therefore $2 \mathrm{ml}$ liquid culture were centrifuged at $4000 \mathrm{rpm}$ for $5 \mathrm{~min}$, washed with $1 \mathrm{ml}$ $50 \mathrm{mM}$ Tris and centrifuged again. Subsequently, the pellet was resolved in $200 \mu \mathrm{l}$ Tris. For sample preparation, an EM S160-3 cupper grid was incubated on a droplet of liquid $C$. difficile culture, or washed cells, for 1 min to allow absorption of cells to the grid's carbon film. The grid was carefully semi-dried with a filter, preventing crystallization of media ingredients on the carbon film. The grid was washed in a droplet of deionized $\mathrm{H}_{2} \mathrm{O}$, filter-dried and negatively stained on a droplet of $1 \%(\mathrm{w} / \mathrm{v})$ uranyl acetate solution for $15 \mathrm{~s}$. Afterwards the grid was completely dried with a filter and analyzed using a Jeol JEM-1011 TEM.

\section{Antibiotic resistance susceptibility tests}

Susceptibility to metronidazole, erythromycin, vancomycin, rifampicin and moxifloxacin was performed using Etest $^{\oplus}$ strips (bioMérieux, Nürtingen, Germany). Grown cells (according the described general growth conditions above) were adjusted with $0.9 \%$ saline to a McFarland standard 1 and swabbed onto Mueller-Hinton agar supplemented with $5 \%$ horse blood and $20 \mathrm{mg} / \mathrm{l} \beta-\mathrm{NAD}^{+}$ (bioMérieux, Nürtingen, Germany). Plates were incubated anaerobically at $37{ }^{\circ} \mathrm{C}$ and MIC breakpoints were read after $48 \mathrm{~h}$. Control strains (Table 1) were included to verify the reproducibility of the test.

\section{DNA extraction, genome sequencing, de novo genome assembly and genome annotation}

For genome sequencing the strains were cultivated anaerobically in Wilkins-Chalgren Anaerobe Broth (Oxoid,

Table 1 Antibiotic resistance patterns of the three $C$. difficile strains

\begin{tabular}{|c|c|c|c|c|c|}
\hline Isolate & Rifampicin & bancomycin & ${ }^{\mathrm{b}}$ Metronidazole & ${ }^{c}$ Moxifloxacin & Erythromycin \\
\hline DSM 27638 & ${ }^{\mathrm{a}} 0.004$ & $S(0.5)$ & $S(0.38)$ & $R(>32)$ & $R(>256)$ \\
\hline DSM 27639 & $S(0.003)$ & $S(0.75)$ & $S(0.25)$ & $S(2)$ & $R(>256)$ \\
\hline DSM 27640 & ${ }^{\mathrm{a}} 0.004$ & $S(0.38)$ & $S(0.25)$ & $R(>32)$ & $R(>256)$ \\
\hline DSM 27543 & $S(0.003)$ & $S(0.5)$ & $S(0.25)$ & $S(2)$ & $R(>256)$ \\
\hline DSM 27147 & ${ }^{\mathrm{a}} 0.004$ & $S(0.5)$ & $S(0.38)$ & $R(>32)$ & $R(>256)$ \\
\hline
\end{tabular}

May be tested for epidemiological purposes only (ECOFF 4 mg/L (EUCAST Clinical Breakpoint Table v. 7.1 .). DSM 27543 (630) and DSM 27147 (R20291) served as controls

MIC given in $\mathrm{mg} / \mathrm{mL}$, $S$ sensitive, $R$ resistant

${ }^{a}$ Not used clinically. May be tested for epidemiological purposes only (ECOFF $0.004 \mathrm{mg} / \mathrm{L}$ ). Vancomycin: $R>2 \mathrm{mg} / \mathrm{L}$. Metronidazole: $\mathrm{R}>2$

${ }^{\mathrm{b}}$ The breakpoints are based on epidemiological cut-off values, which distinguish wild-type isolates from those with reduced susceptibility

${ }^{\mathrm{c}}$ Not used clinically 
Table 2 Genome sequences used in this study

\begin{tabular}{|c|c|c|c|c|c|c|}
\hline Strain & Ribotype & Toxino-type & Genome size & Isolation (year/country) & Accession number & Reference \\
\hline DSM 27638 & 027 & III & $4,229,698$ & 2015/Germany & СР011846.1 & This study \\
\hline DSM $27639^{a}$ & 012 & 0 & $4,263,997$ & 2015/Germany & СР011847.1 & This study \\
\hline DSM $27640^{\mathrm{a}}$ & 027 & III & $4,229,629$ & 2015/Germany & СР011848.1 & This study \\
\hline $630^{\mathrm{a}}$ & 012 & 0 & $4,274,782$ & 1982/Switzerland & СР010905.2 & {$[27]$} \\
\hline$C F 5^{b}$ & 017 & VIII & $4,159,517$ & 1995/Belgium & FN665652.1 & [14] \\
\hline$M 68^{b}$ & 017 & VIII & $4,308,325$ & 2006/Ireland & NC_017175.1 & [14] \\
\hline $\mathrm{CD}_{196}{ }^{\mathrm{C}}$ & 027 & III & $4,110,554$ & 1985/France & NC_013315.1 & {$[49]$} \\
\hline$R 20291^{d}$ & 027 & III & $4,191,339$ & 2006/UK & FN545816.1 & [14] \\
\hline $2007855^{d}$ & 027 & III & $4,179,867$ & 2007/US & FN665654.1 & {$[14]$} \\
\hline $\mathrm{M} 120^{\mathrm{b}}$ & 078 & V & $4,047,729$ & 2007/UK & NC_017174.1 & [51] \\
\hline
\end{tabular}

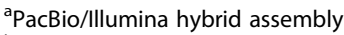

${ }^{\mathrm{b}} 454$ /lllumina hybrid assembly

c454/Sanger sequencing

${ }^{d} 454$ sequencing

Basingstore, United Kingdom) at $37{ }^{\circ} \mathrm{C}$. Genomic DNA was extracted as described previously [23, 24].

Genome sequencing of the $C$. difficile strains was carried out on the PacBio RSII (Pacific Biosciences, Menlo Park, CA) using P5 chemistry. Genome assembly was performed with the RS_HGAP_Assembly.3 protocol included in SMRT Portal version 2.3.0. The chromosomal contigs generated were trimmed, circularized, and adjusted to $d n a A$ as the first gene.

In parallel, genome sequencing of the $C$. difficile strains was carried out on a Genome Analyzer GAIIx (Illumina, San Francisco, CA) in a 112 bp paired-end single-indexed run Quality improvement of the final consensus sequence was performed with the Burrows-Wheeler Aligner (BWA) using bwa aln and bwa sampe [25] mapping the Illumina reads onto the obtained chromosomal contigs from the PacBio sequencing. A final quality score of QV60 was attained. Automated genome annotation was carried out using Prokka [26]. Subsequentially, selenocysteine proteins were annotated manually. Complete genome sequences have been submitted to GenBank under the accession numbers CP011846.1 (DSM 27638), CP011847.1 (DSM 27639) and CP011848.1 (DSM 27640). No extrachromosomal genetic elements were observed within this sequencing study.

\section{Comparative genomics}

The genomes of strains DSM 27638, DSM 27639 and DSM 27640 were compared with finished closed references genomes selected as members of the corresponding RTs, Comparative genomics included three strains belonging to the most virulent RT 027 (non-epidemic strain CD196, the epidemic and highly virulent strain R20291 [14], and the bovine isolate 2,007,855), three other strains belong to recently emerging RTs 017 (CF5 and M68) and RT 078 (M120). The RT 012 is represented by strain 630 [23, 27]. All analyzed strains are listed in Table 2. Orthologous proteins were determined with ProteinOrtho [28] applying default parameters. Circular visualizations and comparisons of shared nucleotide regions of the genomes have been produced

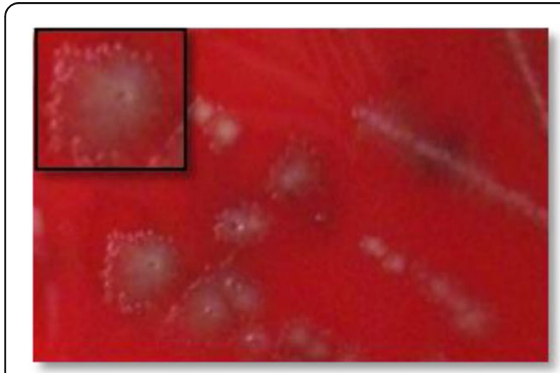

a

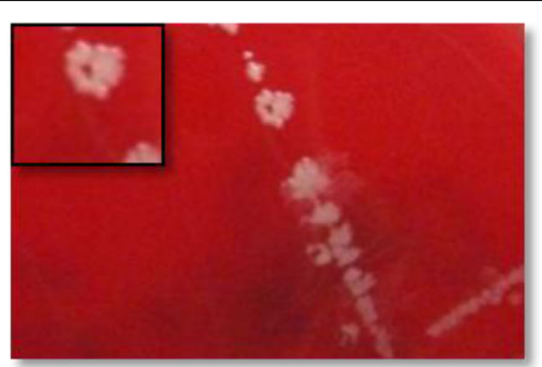

b

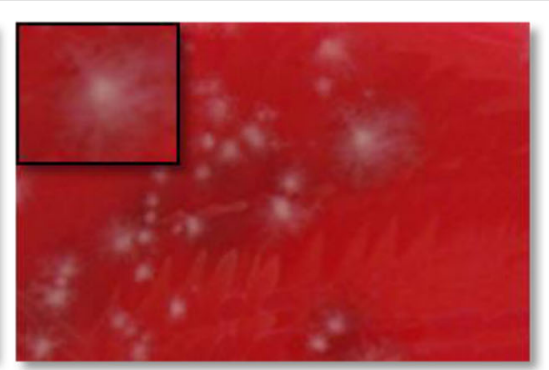

C

Fig. 1 Colony morphology of C. difficile strains. Strains were grown anaerobically $\left(5 \% \mathrm{H}_{2}, 95 \% \mathrm{~N}_{2}\right)$ on commercial Clostridium difficile agar (CLO) for 2 days at $37{ }^{\circ} \mathrm{C}$. DSM 27638 (a) and DSM 27640 (c) show irregular colony shape with shiny surfaces and white color. Colonies from DSM 27640 (c) are more opaque than colonies from DSM 27638 (a). Colony shape of DSM 27639 (b) looks nearly filamentous, colony surface is smooth and the color is cloudy and whitish 


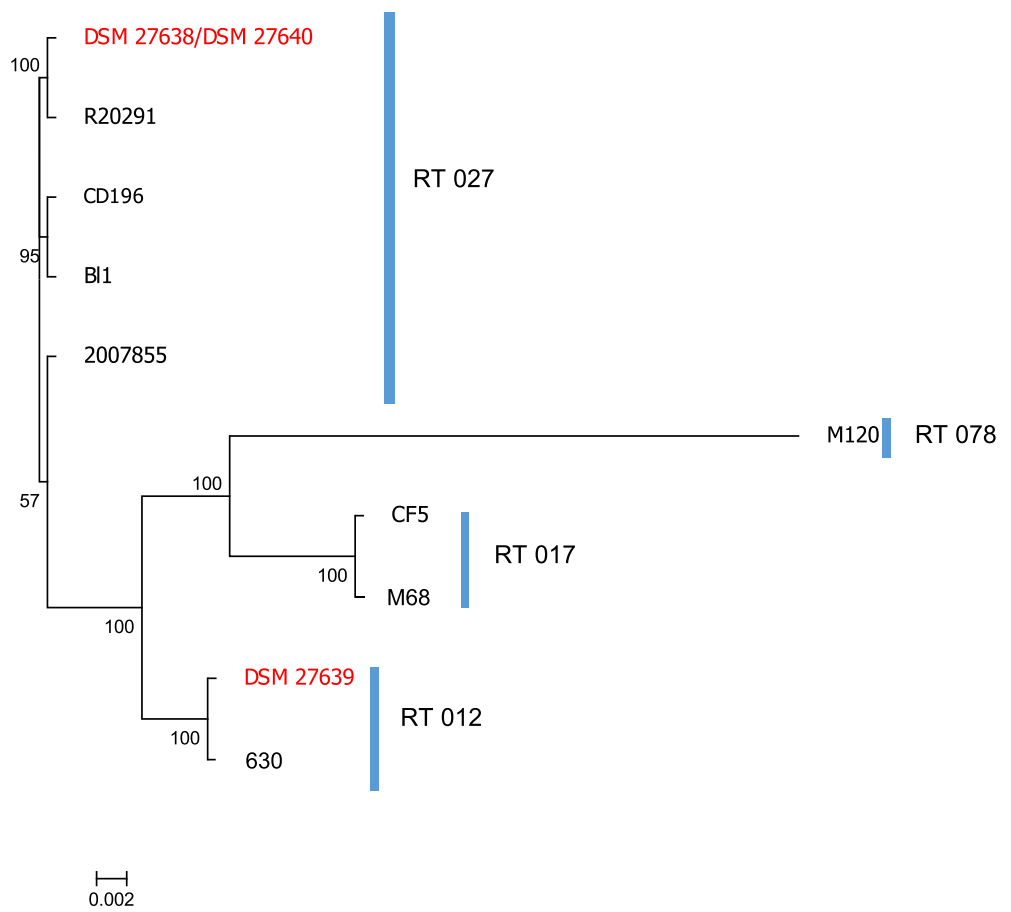

Fig. 2 Whole genome alignment based phylogenomic tree. Strain DSM 27639 clusters with reference strain 630 and strains DSM 27638/40 with all RT 027 strains. The tree has been calculated using Phylomark with default parameters. The clinical isolates are marked in red, due to the extreme low editing distance the node of DSM 27638 and DSM 27640 has been collapsed

with BRIG [29] and linear visualizations with MAUVE [30]. Identification of genomic islands has been done with Island viewer 3 [31]. All identified regions have been manually curated using UniProtKB/Swiss-Prot and TrEMBL database (www.uniprot.org). In detail, comparison of related genome regions have been done with ACT and Artemis [32]. Phylogenomics based on whole genome alignments has been performed by using

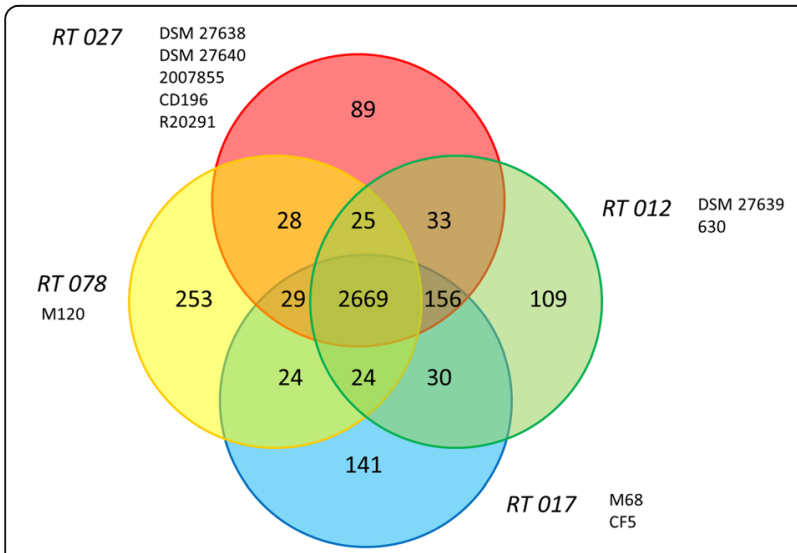

Fig. 3 Core/Pan-genome calculation of the RT 012, RT 017, RT 027, and RT 078. The four ribotypes share a core genome of 2669 genes. The genomes used to generate the protein datasets are indicated. Orthologous proteins have been identified with ProteinOrtho software (Lechner et al., [28])
Phylomark [33]. Synteny analysis and SNP prediction have been performed with nucmer from the mummer program suite [34].

\section{Results and discussion} Phenotypic characterization

Three C. difficile strains (DSM 27638, DSM 27639 and DSM 27640) exhibiting different phenotypes on solid medium have been isolated from one stool sample of a single patient suffering from CDI. The isolate DSM 27639 formed colony types that were white and smooth with clearly defined edges (Fig. 1). The other two isolates DSM 27638 and DSM 27640 had a rougher surface and seemed to spread on the agar plate (Fig. 1). Both isolates mainly differed in color; isolate DSM 27638 was grayish compared to isolate DSM 27640 which appeared rather gray beige. This initial observation indicated that the patient was infected with more than only one $C$. difficile strain. Since it has been reported that multiple infection with pathogens like $C$. difficile occur $[20,21]$, we aimed to more precisely characterize the respective phenotypic and genotypic differences that occurred during this multiple infection.

Toxinotyping has been used for distinguishing $C$. difficile strains. In this regard, toxin $\mathrm{A}$ and $\mathrm{B}$ genes located on the PaLoc are considered to be the major 

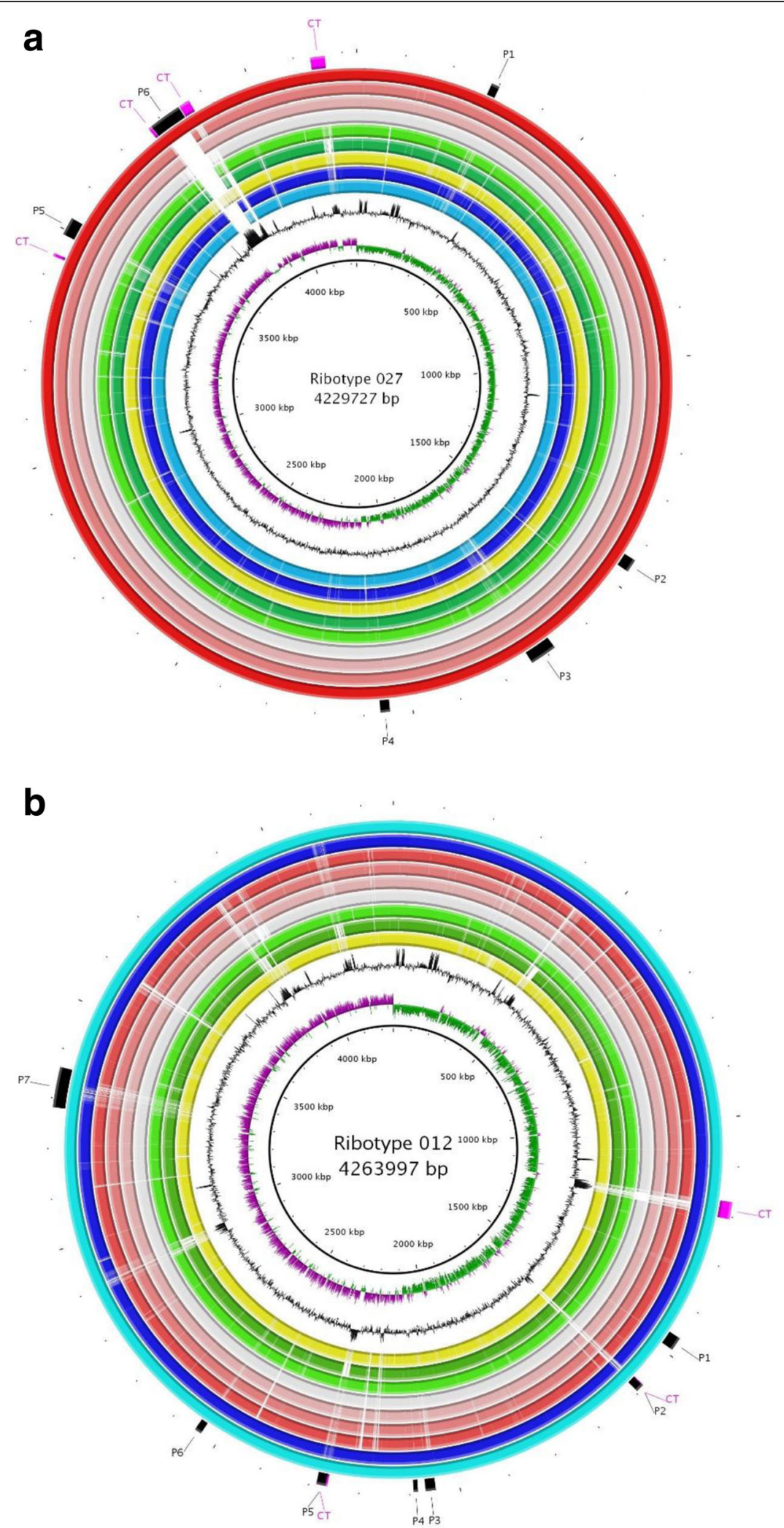

Fig. 4 (See legend on next page.) 
(See figure on previous page.)

Fig. 4 Circular representations of $C$. difficile chromosomes generated with BRIG software. From the outside: circle 1 shows identified mobile elements (black - prophages P1-7, pink - conjugative transposons CT). Circle 2 shows reference genome a strain DSM 27638 / DSM 27640, b strain DSM 27639). The most inner circle represents scale (in kb), second inner - GC skew for reference genome, third - GC content graph. Inner rings represent analyzed genome sequences of red colors ribotype 027 (the most dark red: DSM 27638 then 2,007,855, R20291, and light red 196); blue colors represent 012 ribotype (dark blue: 630, light blue: DSM 27639); green colors represent ribotype 017 (dark green: CF5, light green: M68) and yellow color represent strain M120 belonging to RT 078

virulence factors of $C$. difficile. Sequencing of the complete genomes showed that the PaLocs from all three isolates were intact. As expected, the toxin loci of DSM 27638 and DSM 27640 grouped to toxinotype III, which is observed for strains belonging to RT 027 [35]. The toxin locus of isolate DSM 27639 belonged to toxinotype 0, which correlated with strains grouping into RT 012 [35].

Both RT 027 isolates DSM 27638 and DSM 27640 exhibited the typical antimicrobial susceptibility pattern of this RT, including high resistance against erythromycin and moxifloxacin (Table 1). In contrast, the RT 012 isolate DSM 27639 was susceptible to moxifloxacin. All isolates were susceptible to vancomycin and metronidazole that are antibiotics commonly used in CDI therapy [36].

It has been reported that multiple $C$. difficile strains can co-exist in an in-vitro human gut model although exhibiting different growth rates [37], we investigated the growth behavior of the three isolates under pure culture conditions as well as under co-cultivation conditions. However, the strains showed an identical growth behavior in BHIS under standard conditions (Additional file 1: Figure S1) In addition, all strains had the same maximal sporulation ability shown by comparable amounts of germinated spores on plates after incubation under harsh nutrient starvation (Additional file 2: Figure S2). Co-cultivation of the isolates showed that none of them had either a negative or a positive effect on the growth of any of the other isolates or the growth of reference strain 630 (data not shown). The absence of intra-species competition most likely has supported their co-existence in the patient. However, those in vitro results obviously cannot reflect the complex situation in the human host where nutrients are limiting and the different isolates are challenged by the host immune system and complex gut microbial community.

The clinical isolates DSM 27638, DSM 27639 and DSM 27640 exhibit representative phenotypic features of the toxinotype they belong to which is confirmed by the phylogenetic clustering based on whole genome sequence comparison (Fig. 2). However, phenotypic analysis could not explain the presence of two RTs in one patient.

\section{General genome comparison}

To determine the genomic features correlating with the observed colony phenotypes we performed complete genome sequencing of all three isolates. Their genomes were compared with seven publicly available closed $C$. difficile genomes including reference genomes of four different PCR ribotypes. A whole genome alignment using Phylomark [33] was used to assign strains DSM 27638 and DSM 27640 to RT 027 and the isolate DSM 27639 to RT 012 (Fig. 2). The genome alignment of these isolates and reference strains using MAUVE showed that all $C$. difficile strains share a complete syntenic chromosome interrupted by mobile elements, as it has been observed in other virulent clostridia [38] (Additional file 3: Figure S3).

To determine the core genome orthologous coding sequences (CDS) between all $C$. difficile strains were identified. Thus, we identified a core genome of 2669 CDS shared by all strains (Fig. 3). Consistent with the antibiotic dependent pathogenicity of CDI the core genomes comprises a number of genes assigned to antimicrobial resistances (Additional file 4: Table S1), including the beta-lactamase-inducing penicillin-binding protein BlaR; the quaternary ammonium compoundresistance protein SugE, and the vancomycin/teicoplanin-resistance proteins VanG, VanV and VanW. Resistance of $C$. difficile against the fluoroquinolone moxifloxacin is characteristic for most RT 027 strains and provides them with a selective advantage in comparison to other ribotypes when this antibiotic is used. The historical RT 027 strain CD196 in contrast to recently isolated RT 027 strains is moxifloxacin susceptible [39]. It was already shown that a single point mutation in the DNA gyrase subunit A-encoding gene gyrA of $C$. difficile leads to fluoroquinolone resistance [40]. In contrast to $C$. difficile reference strain 630 and isolate DSM 27639, the RT 027 strain 2,007,855 and the isolates DSM 27638 and DSM 27640 are resistant to fluoroquinolones. Sequence analysis of the GyrA protein confirmed that all moxifloxacin-resistant $C$. difficile strains contain a single transition mutation resulting in the amino acid substitution Thr-82-Ile (Additional file 5: Figure S4) [41].

In addition, strain-specific and ribotype-specific CDS were identified. The locations of regions of genetic difference between the strains are highlighted in the concentric circular chromosome representations of the analyzed ten genomes (Fig. 4). Strain specific genes are often found to be encoded in regions that have been identified as prophages or conjugative transposons (Table 3). 
Table 3 Mobile elements of strains DSM 27638 and DSM 27639

\begin{tabular}{|c|c|c|c|c|}
\hline Start & Stop & $\begin{array}{l}\text { Length } \\
\text { [bp] }\end{array}$ & ORFs & $\begin{array}{l}\text { Mobile element } \\
\text { and gene content }\end{array}$ \\
\hline \multicolumn{5}{|c|}{ DSM 27638} \\
\hline 284,586 & 301,831 & 17,244 & 16 & $\begin{array}{l}\text { PHAGE (P1) (not found in } \\
012 \text { and } 017 \text { ribotypes) }\end{array}$ \\
\hline 398,255 & 402,824 & 4569 & 7 & $\begin{array}{l}\text { ABC transporter, two- } \\
\text { component system, } \\
\text { transposase }\end{array}$ \\
\hline 501,871 & 511,046 & 9229 & 10 & $\begin{array}{l}\text { transposase, hydrolases, } \\
\text { transcriptional regulator, } \\
\text { oligo-1,6-glucosidase, } \\
\text { PTS system transporter } \\
\text { subunit IIABC }\end{array}$ \\
\hline 518,083 & 528,817 & 10,734 & 10 & $\begin{array}{l}\text { lantibiotic resistance, two } \\
\text { two-component systems, } \\
\text { ABC transporter }\end{array}$ \\
\hline 662,517 & 674,897 & 12,380 & 11 & $\begin{array}{l}\text { ABC transporter, two- } \\
\text { component system, } \\
\text { transcriptional regulator }\end{array}$ \\
\hline 934,705 & 938,946 & 4241 & 5 & $\begin{array}{l}\text { lantibiotic resistance two- } \\
\text { component system, two- } \\
\text { component system }\end{array}$ \\
\hline $1,438,093$ & $1,465,397$ & 27,304 & 30 & PHAGE (P2) \\
\hline $1,680,933$ & $1,736,907$ & 55,974 & 69 & $\begin{array}{l}\text { PHAGE (P3) (ribotype } \\
027 \text { specific) }\end{array}$ \\
\hline $2,046,513$ & $2,066,289$ & 19,776 & 6 & $\begin{array}{l}\text { PHAGE (P4) (ribotype } \\
027 \text { specific) }\end{array}$ \\
\hline $3,441,029$ & $3,447,002$ & 5973 & 6 & conjugative transposon \\
\hline $3,478,606$ & $3,491,098$ & 12,492 & 8 & $\begin{array}{l}\text { integrase, chromosome } \\
\text { segregation ATPase }\end{array}$ \\
\hline $3,489,961$ & $3,527,152$ & 37,191 & 26 & $\begin{array}{l}\text { PHAGE (P5) (ribotype } \\
027 \text { specific) }\end{array}$ \\
\hline $3,769,213$ & $3,775,340$ & 6127 & 7 & conjugative transposon \\
\hline $3,775,480$ & $3,844,734$ & 69,254 & 54 & $\begin{array}{l}\text { PHAGE (P6) (DSM } 27638 \\
\text { specific) }\end{array}$ \\
\hline $3,846,878$ & $3,869,593$ & 22,715 & 22 & conjugative transposon \\
\hline $4,100,257$ & $4,105,353$ & 5096 & 5 & $\begin{array}{l}\text { ABC transporter, two- } \\
\text { component system }\end{array}$ \\
\hline $4,134,037$ & $4,164,119$ & 30,082 & 34 & $\begin{array}{l}\text { conjugative transposon, } \\
\text { multidrug resistance } \\
\text { protein (ribotype } 027 \\
\text { specific) }\end{array}$ \\
\hline DSM 2763 & & & & \\
\hline $1,170,734$ & $1,205,103$ & 34,369 & 33 & $\begin{array}{l}\text { conjugative transposon, } \\
\text { lantibiotic ABC transporter } \\
\text { (DSM } 27639 \text { specific) }\end{array}$ \\
\hline $1,461,445$ & $1,488,797$ & 27,352 & 30 & PHAGE (P1) \\
\hline $1,578,539$ & $1,595,263$ & 16,724 & 17 & $\begin{array}{l}\text { conjugative transposon } \\
\text { (DSM } 27639 \text { specific) }\end{array}$ \\
\hline $1,580,053$ & $1,595,960$ & 15,907 & 9 & $\begin{array}{l}\text { PHAGE (P2) (DSM } 27639 \\
\text { specific) }\end{array}$ \\
\hline
\end{tabular}

Table 3 Mobile elements of strains DSM 27638 and DSM 27639 (Continued)

\begin{tabular}{|c|c|c|c|c|}
\hline Start & Stop & $\begin{array}{l}\text { Length } \\
\text { [bp] }\end{array}$ & ORFs & $\begin{array}{l}\text { Mobile element } \\
\text { and gene content }\end{array}$ \\
\hline $2,047,626$ & $2,068,044$ & 20,418 & 7 & $\begin{array}{l}\text { PHAGE (P3) (ribotype } \\
012 \text { specific) }\end{array}$ \\
\hline $2,083,042$ & $2,091,718$ & 8676 & 9 & PHAGE (P4) \\
\hline $2,263,687$ & $2,285,988$ & 22,301 & 26 & $\begin{array}{l}\text { conjugative transposon } \\
\text { (DSM } 27639 \text { specific) }\end{array}$ \\
\hline $2,267,275$ & $2,285,716$ & 18,441 & 11 & $\begin{array}{l}\text { PHAGE (P5) (DSM } 27639 \\
\text { specific) }\end{array}$ \\
\hline $2,536,302$ & $2,550,493$ & 14,191 & 23 & $\begin{array}{l}\text { PHAGE (P6) (ribotype } \\
012 \text { specific) }\end{array}$ \\
\hline $3,282,029$ & $3,362,995$ & 80,966 & 84 & $\begin{array}{l}\text { PHAGE (P7) (DSM } 27639 \\
\text { specific) }\end{array}$ \\
\hline
\end{tabular}

${ }^{a}$ DSM 27,640 is not included because it is identical to DSM 27638 in all elements. All mobile elements have been predicted by IslandViewer 3 , PHASTER and have been manually curated

The overall similarity strongly underlines that RT 027 strains are closely related. Sequence data show that six genetic regions (two transposons and four prophages P1, P3, P4, and P5) are unique to the RT 027. The biggest difference observed in the genome of DSM 27638 is the presence of a predicted prophage integrated at 3.78 to 3.84 Mbp flanked by conjugative transposons (Fig. 4, Table 3). Interestingly, there is a conjugative transposon present at position 3.84 to $3.87 \mathrm{Mbp}$ shared by the strains DSM 27638 and DSM 27640 as well as strain R20291 but not by the RT 027 reference strain CD196. This indicates that the four strains might share a common history starting at a RT 027 ancestor that did not contain the conjugative transposon. The ancestor of strains DSM 27638, DSM 27640 and R20291 might have acquired the transposon locus, which in the ancestor of strains DSM 27638 and DSM 27640 was the integration locus of a prophage.

Sequence comparison of two RT 012 genomes (630 (DSM 27543) and DSM 27639) revealed a high degree of synteny except for those regions that encode mobile elements (Additional file 3: Figure S3). A circular BLAST based comparison of the DSM 27639 genome focused on the RT 012 genomes revealed that the difference between both genomes correlates directly to predicted prophages and conjugative transposons (Fig. 4 b). Two prophages (P3 and P5) and five transposons are shared exclusively by the genomes of the strains DSM 27639 and 630 and might therefore be acquired by an RT 012 ancestor. The remaining two prophages ( $\mathrm{P} 1$ and $\mathrm{P} 4)$ are specific for strain DSM 27639. One prophage region is shared by all nine analyzed strains.

Comparative genomics revealed that the biggest difference of the strains isolated from the same patient to the reference genomes is the acquisition or loss of 
prophages and conjugative transposons, which fits to the observations of Hargreaves et al. and Mullany et al. [18, 42]. Thus we conclude that the strain-specific genes are a result of acquisition of mobile elements, which indicates the importance of these elements for the emergence of virulence. In contrast to the prophage regions, all conjugative transposons correlate with GC-content variations compared to the average GC-content (see Fig. 4). This indicates that the acquisition of the transposons are a more recent event and thus the forces of amelioration of newly acquired genome regions (described in [43] and references therein) to the host genome have had less time to operate.

\section{In infectio genome dynamics of DSM 27638 and DSM 27640}

The genome sequences of the two RT 027 isolates DSM 27638 and DSM 27640 are almost identical (Additional file 6: Figure S5), which suggest a clonal history of them within the patient $[14,44]$. The isolates encode the same number of predicted proteins and differ by only 69 bp in size (Table 2, Fig. 5). A whole genome BLAST comparison of the genomes revealed the differences within six genome regions five of them being found within intergenic regions (Table 4). Two loci represent imperfect inverted repeats upstream of operons, a motif which has been found in Salmonella enterica as regulatory element where the inverted repeat regulates the downstream operon upon inversion [45]. Johnson described the regulation as a reversible flip/flop mechanism. Three inverted loci flanked by inverted repeats have been described as well as a difference between the originally published of the $C$. difficile genome $630 \Delta \mathrm{erm}$ and a high quality re-sequenced version in Dannheim at el. [27] indicating a reversible nature of these genomic elements. However, a comparison of the reverse complement sequence from isolate DSM 27638 with the sequence of DSM 27640 revealed that the regions differ in six base positions between the two strains. In contrast to the $630 \Delta \mathrm{erm}$ strains the locus differs between strains DSM 27638 and DSM 27640 not only in a reversible inversion. The locus is located upstream of the first CDS of the late flagellum genes and has been investigated in detail by Anjuwon Foster et al. [46] who named the regulatory element as flagellum switch. The sequence of strain DSM 27638 is identical to the $154 \mathrm{bp}$ sequence described for RT 027 in contrast to the DSM 27640 version that contains additionally 4 bp. In contrast, the second inverted repeat, which is located upstream of a diguanylate cyclase, exhibits no difference compared to the reverse complement sequence of the corresponding locus of strain DSM 27640. This observation and the possibility that this kind of inverted repeat may be reversibly inverted $[45,47]$ challenges the hypothesis that the two genome regions really can be considered as different. Furthermore, the operons located adjacent to the inverted repeat encode transporters where a possible contribution to a macroscopic visible strain difference is at least not obvious. The most prominent sequence difference between the two isolates is generated by the insertion of eight instances of an octamer repeat-unit in isolate DSM 27638 at position 594,943 to 595,006 . This 64 bp insertion is responsible for almost the complete size difference of $69 \mathrm{bp}$ of the two genomes (Table 2). As a result DSM 27638 contains 21 repeat-units and DSM 2764013 repeat-units at the corresponding locus. The repetitive region is located within the intergenic region of a locus that encodes several genes assigned to spore surface components. However, although it is possible

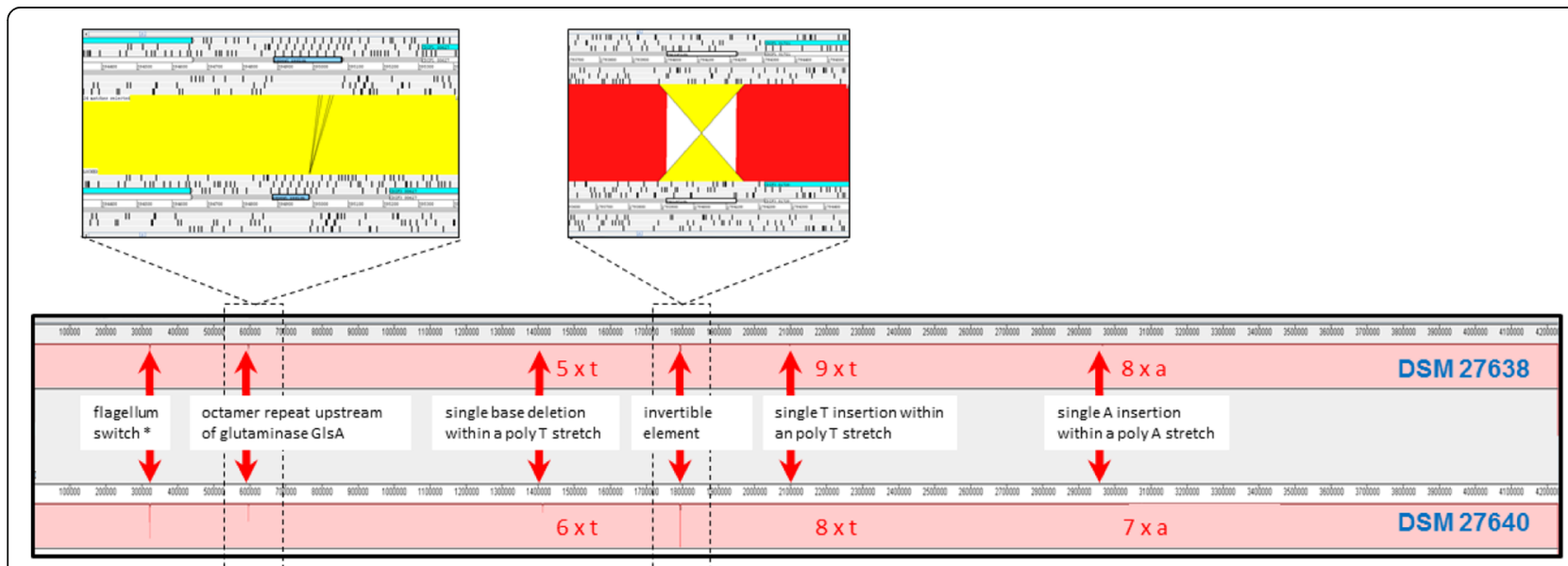

Fig. 5 Sequence differences of C. difficile isolates DSM 27638 and DSM 27640. Three regions out of six are point mutations. The flagellum switch region is described in detail in Fig. 7. The octamer repeat region and an invertable element are presented in detail 
Table 4 Sequence differences of C. difficile isolates DSM 27638 and DSM 27640

\begin{tabular}{|c|c|c|}
\hline DSM 27638 & DSM 27640 & Description \\
\hline 321,766 to 321,922 & 321,766 to 321,918 & $\begin{array}{l}\text { six base variations within the flagellum switch, an invertible element downstream } \\
\text { of a c-di-GMP riboswitch within the 5'UTR of the flgB-gene }\end{array}$ \\
\hline 594,884 to 595,051 & 594,880 to 594,983 & $\begin{array}{l}\text { octamer repeat upstream of glutaminase GlsA, DSM } 27638 \text { contains eight } \\
\text { additional instances }\end{array}$ \\
\hline Gap at 1,411,589 & $\mathrm{T}$ at $1,411,522$ & $\begin{array}{l}\text { single base deletion within a poly T stretch generates a disfunctional signal } \\
\text { peptidase Sip3 gene in DSM } 27638\end{array}$ \\
\hline $1,793,982$ to $1,794,196$ & $1,793,915$ to $1,794,129$ & $\begin{array}{l}\text { invertible element upstream of signaling protein (CDIF27640_01720 resp. } \\
\text { CDIF27638_01720). The reverse complement of the DSM } 27638 \text { sequence } \\
\text { is identical to the DSM } 27640 \text { sequence }\end{array}$ \\
\hline T at 2,096,993 & Gap 2,096,925 & $\begin{array}{l}\text { single T insertion within an poly } T \text { stretch, upstream of stage } V \text { sporulation } \\
\text { protein S SpoVS }\end{array}$ \\
\hline A at $2,964,477$ & . Gap 2,964,408 & $\begin{array}{l}\text { single A insertion within a poly A stretch, upstream of a purine riboswitch regulated } \\
\text { permease (CDIF27638_02797 resp. CDIF27640_02796) }\end{array}$ \\
\hline
\end{tabular}

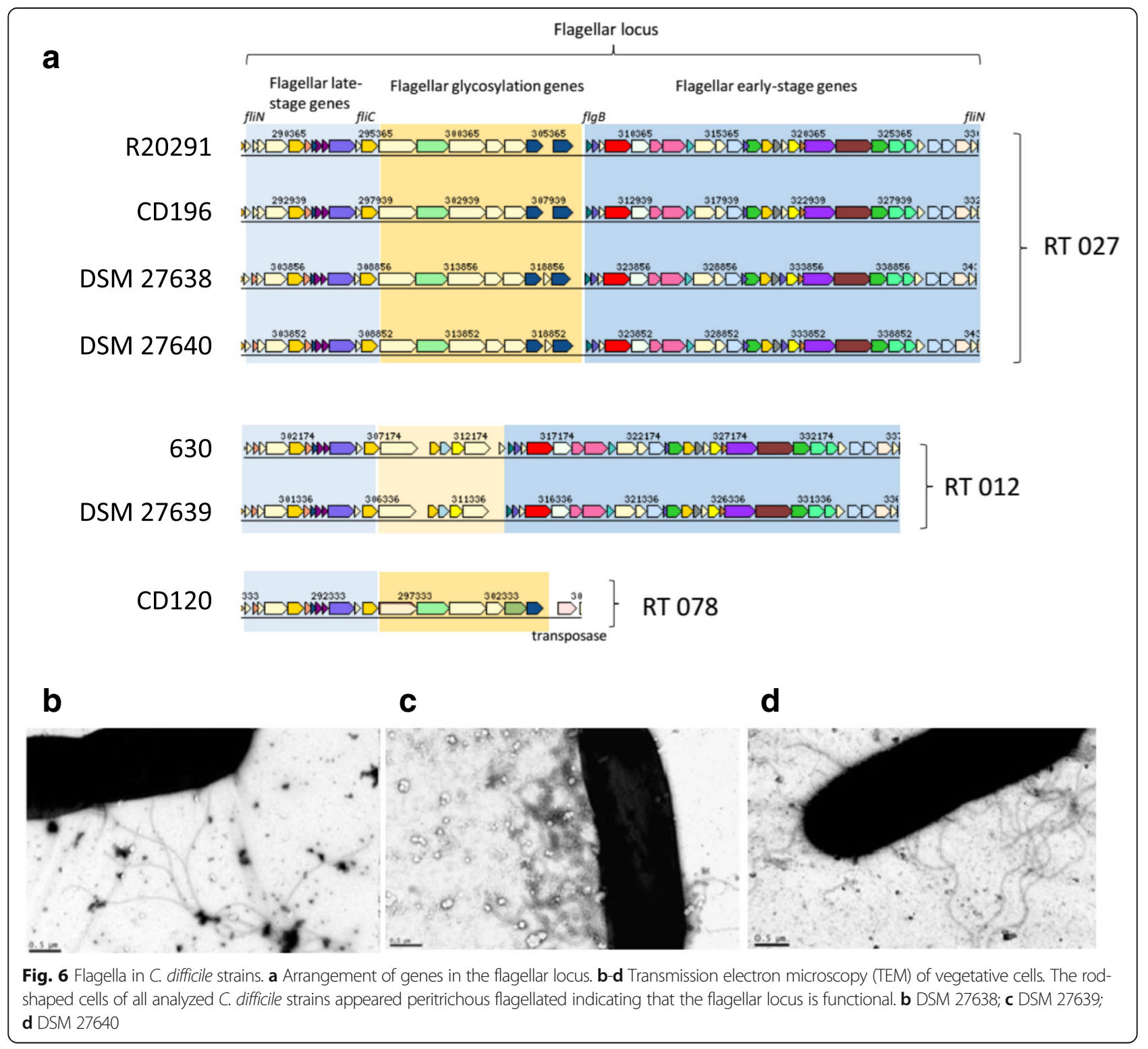


that a modification of the regulation of spore surface components might result in the observed phenotypic differences a comparative investigation of the sporulation behavior and the viability of the spores revealed no significant differences between the three isolates (Additional file 2: Figure S2). Since the two isolates are - in contrast to the well investigated reference strain 630 - not yet genetically accessibility, a systematic investigation of the multiple repeat region and its putative contribution to the observed differences of growth phenotypes is not possible to date. The remaining genome sequence differences are three single base insertion, two of them located in intergenic regions and only one impacts an encoding signal peptidase.

\section{The fli locus}

The fli locus encodes the flagellum of $C$. difficile, which results in motile peritrichous $C$. difficile cells [48]. A genome analysis of the $f l i$ loci of the three isolates and comparisons with RT 027 and RT 012 reference strains as well as with a non-motile RT 078 control strain confirmed that all structural genes necessary to encode a functional flagellum are present in all three isolates, a

DSM 27638

DSM 27640

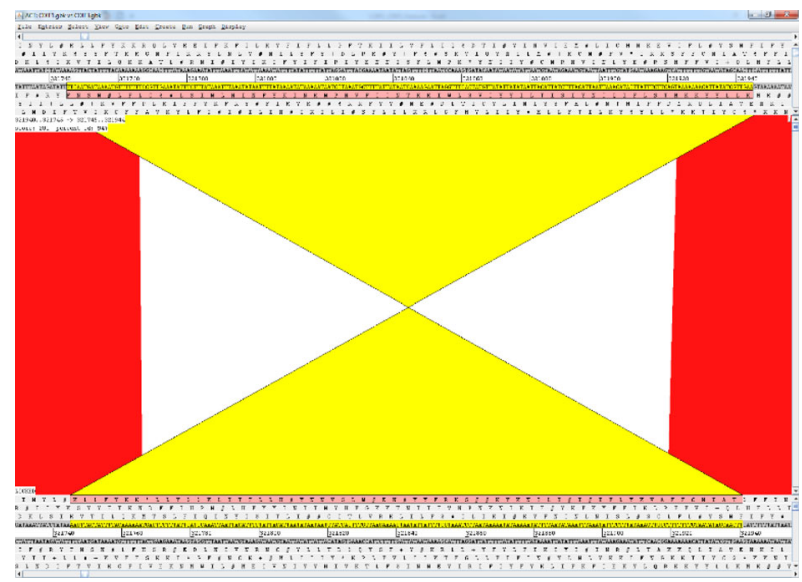

b

DSM 27638 AGGCAACTTTATAAAG--AAAT-ATTTAAATTTATATTAAAATATTTTATATTTTTTATTA | | ||||| | |||| | || | |||| ||||| | || | || | || |

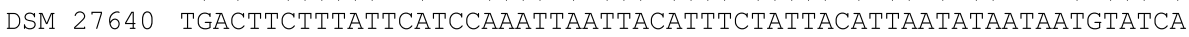

DSM 27638 GGATT-TACGAAAA-TAATATTAGTTTTCTTAATCCAAAGTGATACAATATAAтAтATTA DSM 27640 CTTTGGTAAGAAAACTAATATTATTTTCGTAAATCCTAA-TAAAAATATAAAA-ATATTT

DSM 27638 ATGTAATAGAAATGAATTAATTTGTATGAATAAAGAATCA

||||| |||| ||| || ||| | | ||||||| | |

DSM 27640 ---TAATATAAATTTAAATA-TTTCTTT--ATAAAGTTGCC

\section{C}

DSM 27638 TGACTTCTTTATTCATACAAATTAATTACATTTCTATTACATTAATATATTATATTGTAT |||||||||||||||| ||||||||||||||||||||||||||||||||||| ||| ||||| DSM 27640 TGACTTCTTTATTCATCCAAATTAATTACATTTCTATTACATTAATATA--ATAATGTAT

DSM 27638 CACTTTGGATTAAGAAAACTAATATTATTTTCGTAAATCCTAATAAAAATATAAAA-TAT |||||||| |||||||||||||||||||||||||||||||||||||||||||||||||||| ||| DSM 27640 CACTTTGG--TAAGAAAACTAATATTATTTTCGTAAATCCTAATAAAAATATAAAAATAT

DSM 27638 TTTAATATAAATTTAAATATTTCTTTATAAAGTTGCC || || || || || || ||||||||||||||||||||||||

DSM 27640 TTTAATATAAATTTAAATATTTCTTTATAAAGTTGCC

Fig. 7 Comparison of upstream region of flgB in strains DSM 27638 and DSM 27640. a Pairwise comparison of the C. difficile DSM 27638 and DSM 27640 region of inversion displayed using the Artemis Comparison Tool (ACT; http://www.sanger.ac.uk/science/tools/artemis-comparison-tool-act). The red and yellow bars indicate regions of similarity with red bars indicating corresponding regions that are oriented similarly and yellow bars indicating regions oriented in opposite directions; b Alignment of region from DSM 27638 (321766-321,922) and DSM 27640 (321766-321,918); c Alignment of complement/reverse version from DSM 27640 with DSM 27638 original sequence 
which could be confirmed via TEM (Fig. 6). Note that that all amino acid sequences of the fli-gene products of strains DSM 27638 and DSM 27640 are identical. Since the intergenic regulatory region upstream of the early stage $f l i$ genes is one of only three regional sequence differences identified in the complete genome sequences of DSM 27638 and 27,640 we performed additional phenotypic investigations to verify the expression of a functional flagellum (Fig. 7). In contrast to the nonmotile RT 078 control strain, both RT 027 isolates as well as the RT 012 isolate DSM 27639 and the respective control isolates R20291 (RT 027) and 630 (DSM 27543, RT 012) exhibited a spreading diffuse growth indicative for active motility (Additional file 7: Figure S6). Thus the impact of the described genome difference on the initially observed growth phenotype (Fig. 1) remains unsolved. However, it has been reported that the flagellum can have an impact on the adherence to intestinal mucosa and might eventually also influence growth on solid surfaces such as agar plates $[46,49,50]$. Thus it is tempting to speculate that the sequence difference within the fli locus contributes to the observed growth phenotype of the three patient isolates.

\section{Conclusion}

The analysis of three phenotypic diverse $C$. difficile isolates that were isolated simultaneously from a stool sample of a diarrheic patient confirmed that multiple and isochronal infections with different RTs occur. The phenotypic and genetic characterization could not give an answer which strain (if that is a case) caused the CDI since all three isolates harbor apparently an intact complete PaLoc encoding the $t c d A$ and $t c d B$ toxin genes and there are no obvious phenotypic advantages showing that one isolate distinctly differs from the others. However, our sequence-based analysis gave insights into genome evolution in micro- and macroscale, as well as in in infectio adaptation. The genome history of the three analyzed isolates has been tracked by a comparative genome analysis. The acquisition/loss of prophage and conjugative transposons is most impressive. The observation that strains of different RTs within single infection have exchanged genetic material in form of mobile genetic elements indicates that genome variation might be as well an effect of a community-based maintenance of a common pan-genome which would be separate genomic adaptations from evolutionary events.

Apparently, inversion events of intergenic regions correlate to phenotypic variation. An in-depth analysis of two isolates from RT 027 indicate an in infection strain adaptation. Thus genome modification events which lead to phenotypic diversification and in longterm to the evolution of new strains can be observed in a single infection event.

\section{Additional files}

Additional file 1: Figure S1. Growth curves in BHIS of DSM 27638, DSM 27639 and DSM 27640. The isolates were grown in brain heart infusion medium containing $0.5 \%(\mathrm{~W} / \mathrm{V})$ yeast extract und $0.03 \%(\mathrm{w} / \mathrm{v})$ $\mathrm{L}$-cysteine. All isolates have the same growth rate under laboratory conditions as shown as the means of three replicates with standard deviation. (DOCX $55 \mathrm{~kb}$ )

Additional file 2: Figure S2. Sporulation assay of DSM 27638, DSM 27639 and DSM 27640 on ChromID plates after 5 days incubation on BHIS. All three isolates show a comparable count of spores that germinated on the plate. (DOCX $275 \mathrm{~kb}$ )

Additional file 3: Figure S3. Mauve whole genome alignments of ten linearized C. difficile strains from four different PCR ribotypes. In this alignment process locally collinear blocks (LCBs) were generated. Boxes with identical colors represent $L C B$, indicating homologous DNA regions shared between the chromosomes without sequence rearrangement. Lines collate aligned segments between genomes. The vertical bars denote the conservation level, and upward and downward orientations relative to the genome line indicates collinear and inverted regions, respectively. The only non syntenic region of the genomes is indicated by the red box. Sequences outside colored blocks do not have homologues in the other genome. (DOCX $419 \mathrm{~kb}$ )

Additional file 4: Table S1. Genes assigned to resistance. (DOCX 16 kb) Additional file 5: Figure S4. DNA gyrase subunit A alignment. The resistance phenotype is encoded by the amino acid substitution at the position 82 of GyrA. (DOCX $33 \mathrm{~kb}$ )

Additional file 6: Figure S5. Genome comparison of $C$. difficile isolates DSM 27638 and DSM 27640. The genome share a complete highly conserved chromosome reflected by the main diagonal. The repeats are depicted by the blue dots evenly distributed within the graph. The comparison has been calculated with Mummer 3 using default parameters. (DOCX $603 \mathrm{~kb}$ )

Additional file 7: Figure S6. Motility assay of C. difficile isolates. RT 012 strains (630; DSM 27639), RT 027 strains (R20291; DSM 27640; DSM 27638) and non-motile RT 078 (DSM 29747) as a reference were analyzed on 0.3\% $\mathrm{BH}$-agar. Following 1 to 2 days of post inoculation, the diffusion radius was monitored in semi-solid hungate tubes (A) and on semi-solid agar plates (B), respectively. Note, all strains produced gas. (DOCX $11038 \mathrm{~kb}$ )

\section{Abbreviations}

AAD: Antibiotic associated diarrhea; BEAST: Bayesian evolutionary analysis by sampling trees; $\mathrm{BHI}$ : Brain heart infusion; BHIS: Brain heart infusion supplemented; BWA: Burrows-Wheeler Aligner; C: Clostridioides; CDI: C. difficile infection; CDIFF: C. difficile agar; CDS: Coding sequences; CFU: Colony forming units; CLO: Clostridium difficile agar; COS: Columbia agar with 5\% sheep blood; HGT: Horizontal gene transfer; MRSA: Methicillin-resistant Staphylococcus aureus; PaLoc: Pathogenicity locus; RT: Ribotype;

TEM: Transmission Electron Microscopy

\section{Acknowledgements}

We are grateful to Ines Oehmig, Carolin Pilke, Simone Severitt and Nicole Heyer for excellent technical assistance. We acknowledge the students Johannes Gibhard and Cedric Blötz for the help with some experiments. We thank Dr. Michael Hoppert for expert support and advice with the electron micrographs.

\section{Funding}

This work was funded by the Federal State of Lower Saxony, Niedersächsisches Vorab (WWZN2889/3215). We acknowledge support by the Open Access Publication Funds of the Goettingen University.

\section{Availability of data and materials}

All used strains are deposited and available at the Leibniz Institute DSMZGerman collection of Microorganisms and Cell Cultures using the DSM numbers 27,638, 27,639 and 27,640. All genome sequences are available at GenBank with the accession numbers CP011846.1 (DSM 27638), CP011847.1 (DSM 27639) and CP011848.1 (DSM 27640). 


\section{Authors' contributions}

UG designed research, provided strains, wrote manuscript, EB analyzed data, wrote manuscript, KG performed sporulation experiments, antibiotic assay, co-cultivation assays and analyzed data, JS performed motility assays, made TEM images and analyzed flagellum locus, TR and BB generated genome assemblies, worked on the primary sequence and wrote manuscript, CS did long read sequencing, DW performed motility assays, AP generated high quality genome annotations, CC performed whole genome comparisons and identified mobile genomic elements, WB performed sporulation assays, JO designed research and wrote manuscript, $\mathrm{OZ}$ isolated strains and did growth experiments, RD designed research and wrote manuscript, HL designed research, analyzed genomic data and wrote manuscript. All authors interpreted the results and reviewed and approved the final manuscript.

\section{Ethics approval and consent to participate}

Not applicable, the study does not contain human subjects, human material, or human data.

The stool sample of the respective patient was collected and processed within routine diagnosis. Therefore, it was collected as part of standard care and not for purposes of this study. For this reason and since no patientspecific data are provided in this study, ethical clearance was not necessary.

\section{Consent for publication}

Not applicable, the study does not contain personal data.

\section{Competing interests}

The authors declare that they have no competing interests.

\section{Publisher's Note}

Springer Nature remains neutral with regard to jurisdictional claims in published maps and institutional affiliations.

\section{Author details}

'Institute for Medical Microbiology, University Medical Center Göttingen, Göttingen, Germany. ${ }^{2}$ Department of Genomic and Applied Microbiology \& Göttingen Genomics Laboratory, Institute of Microbiology and Genetics, Georg-August-University, Göttingen, Germany. ${ }^{3}$ Leibniz Institute DSMZ-German Collection of Microorganisms and Cell Cultures, Braunschweig, Germany. ${ }^{4}$ German Center for Infection Research (DZIF), Partner Site Hannover-Braunschweig, Braunschweig, Germany.

\section{Received: 31 July 2017 Accepted: 6 December 2017}

\section{Published online: 02 January 2018}

\section{References}

1. Hensgens MPM, Keessen EC, Squire MM, Riley TV, Koene MGJ, de Boer E, et al. Clostridium difficile infection in the community: a zoonotic disease? Clin Microbiol Infect. 2012;18:635-45.

2. Barbut F, Lalande V, Beaugerie L, Eckert C. Clostridium difficile-associated diarrhea. Hepato-Gastro. 2013;20:111-22.

3. Hryckowian AJ, Pruss KM, Sonnenburg JL. The emerging metabolic view of Clostridium difficile pathogenesis. Curr Opin Microbiol. Elsevier Ltd. 2017;35:42-7.

4. Miller BA, Chen LF, Sexton DJ, Anderson DJ. Comparison of the burdens of hospital-onset, healthcare facility-associated Clostridium difficile infection and of healthcare-associated infection due to methicillin-resistant Staphylococcus aureus in community hospitals. Infect Control Hosp Epidemiol. 2011;32:387-90.

5. Balabanova Y, Gilsdorf A, Buda S, Burger R, Eckmanns T, Gärtner B, et al. Communicable diseases prioritized for surveillance and epidemiological research: results of a standardized prioritization procedure in Germany, 2011. PLoS One. 2011;6:1-7.

6. Wilcox MH. Overcoming barriers to effective recognition and diagnosis of Clostridium difficile infection. Clin Microbiol Infect. European Society of Clinical Microbiology and Infectious Diseases. 2012;18:13-20.

7. Knight DR, Elliott B, Chang BJ, Perkins TT, Riley TV. Diversity and evolution in the genome of Clostridium difficile. Clin Microbiol Rev. 2015;28:721-41.

8. Lessa FC, Gould CV, Clifford McDonald L. Current status of Clostridium difficile infection epidemiology. Clin Infect Dis. 2012;55:65-70.

9. Ghose C. Clostridium difficile infection in the twenty-first century. Emerg Microbes Infect. 2013;2:e62.
10. Steglich M, Nitsche A, von Müller L, Herrmann M, Kohl TA, Niemann S, et al. Tracing the spread of Clostridium difficile ribotype 027 in Germany based on bacterial genome sequences. PLoS One. 2015;10:e0139811.

11. Masri L, Branca A, Sheppard AE, Papkou A, Laehnemann D, Guenther PS, et al. Host-pathogen coevolution: the selective advantage of Bacillus thuringiensis virulence and its cry toxin genes. PLoS Biol. 2015;13:e1002169.

12. Zdziarski J, Brzuszkiewicz E, Wullt B, Liesegang H, Biran D, Voigt B, et al. Host imprints on bacterial genomes-rapid, divergent evolution in individual patients. PLoS Pathog. 2010;6:e1001078.

13. Zhang L, Dong D, Jiang C, Li Z, Wang X, Peng Y. Insight into alteration of gut microbiota in Clostridium difficile infection and asymptomatic C. difficile colonization. Anaerobe. 2015;34:1-7.

14. He M, Sebaihia M, Lawley TD, Stabler RA, Dawson LF, Martin MJ, et al. Evolutionary dynamics of Clostridium difficile over short and long time scales. Proc Natl Acad Sci U S A. 2010;107:7527-32.

15. Sebaihia M, Wren BW, Mullany P, Fairweather NF, Minton N, Stabler R, et al. The multidrug-resistant human pathogen Clostridium difficile has a highly mobile, mosaic genome. Nat Genet. 2006;38:779-86.

16. Stabler RA, Dawson LF, Phua LTH, Wren BW. Comparative analysis of BI/ NAP1/027 hypervirulent strains reveals novel toxin B-encoding gene (tcdB) sequences. J Med Microbiol. 2008:57:771-5.

17. Braun V, Hundsberger T, Leukel $P$, Sauerborn M, Von Eichel-Streiber $C$. Definition of the single integration site of the pathogenicity locus in Clostridium difficile. Gene. 1996;181:29-38.

18. Mullany P, Allan E, Roberts AP. Mobile genetic elements in Clostridium difficile and their role in genome function. Res Microbiol. Elsevier Masson SAS. 2015;166:361-7.

19. Brzuszkiewicz E, Thürmer A, Schuldes J, Leimbach A, Liesegang H, Meyer $F-D$, et al. Genome sequence analyses of two isolates from the recent Escherichia coli outbreak in Germany reveal the emergence of a new pathotype: Entero-Aggregative-Haemorrhagic Escherichia Coli (EAHEC). Arch Microbiol. 2011;193:883-91.

20. Eyre DW, Cule ML, Griffiths D, Crook DW, Peto TEA, Walker AS, et al. Detection of mixed infection from bacterial whole genome sequence data allows assessment of its role in Clostridium difficile transmission. PLoS Comput Biol. 2013;9:e1003059.

21. Balmer O, Tanner M. Prevalence and implications of multiple-strain infections. Lancet Infect Dis. 2011:11:868-78.

22. Burns DA, Heeg D, Cartman ST, Minton NP. Reconsidering the Sporulation characteristics of Hypervirulent Clostridium difficile BI/NAP1/027. PLoS One. 2011;6:e24894.

23. Riedel T, Bunk B, Thuermer A, Sproer C, Brzuszkiewicz EB, et al. Genome Resequencing of the virulent and multidrug-resistant reference strain Clostridium difficile 630. Genome Announc. 2015;3:15-6.

24. Riedel T, Bunk B, Wittmann J, Thürmer A, Spröer C, Gronow S, et al. Complete genome sequence of the Clostridium difficile type strain DSM 1296T. Genome Announc. 2015:3:3-4.

25. Li H, Durbin R. Fast and accurate short read alignment with burrowswheeler transform. Bioinformatics. 2009:25:1754-60.

26. Seemann T. Prokka: rapid prokaryotic genome annotation. Bioinformatics. 2014;30:2068-9.

27. Dannheim H, Riedel T, Neumann-Schaal M, Bunk B, Schober I, Spröer C, et al. Manual curation and reannotation of the genomes of Clostridium difficile 630Aerm and C. difficile 630. J Med Microbiol. Microbiology Society. 2017;66:286-93.

28. Lechner M, Findeiss S, Steiner L, Marz M, Stadler PF, Prohaska SJ. Proteinortho: detection of (co-)orthologs in large-scale analysis. BMC Bioinformatics. BioMed Central Ltd. 2011;12:124.

29. Alikhan N-F, Petty NK, Ben Zakour NL, Beatson SA. BLAST ring image generator (BRIG): simple prokaryote genome comparisons. BMC Genomics. BioMed Central Ltd. 2011;12:402.

30. Darling AE, Mau B, Perna NT. progressiveMauve: multiple genome alignment with gene gain, loss and rearrangement. PLoS One. 2010;5:e11147.

31. Dhillon BK, Laird MR, Shay JA, Winsor GL, Lo R, Nizam F, et al. IslandViewer 3: more flexible, interactive genomic island discovery, visualization and analysis. Nucleic Acids Res. 2015;43:104-8.

32. Carver T, Berriman M, Tivey A, Patel C, Böhme U, Barrell BG, et al. Artemis and $A C T$ : viewing, annotating and comparing sequences stored in a relational database. Bioinformatics. 2008;24:2672-6.

33. Sahl JW, Matalka MN, Rasko DA. Phylomark, a tool to identify conserved phylogenetic markers from whole-genome alignments. Appl Environ Microbiol. 2012;78:4884-92. 
34. Kurtz S, Phillippy A, Delcher AL, Smoot M, Shumway M, Antonescu C, et al. Versatile and open software for comparing large genomes. Genome Biol. 2004;5:R12.

35. Rupnik M, Brazier JS, Duerden BI, Grabnar M, Stubbs SLJ. Comparison of toxinotyping and PCR ribotyping of Clostridium difficile strains and description of novel toxinotypes. Microbiology. 2001;147:439-47.

36. Aslam S, Hamill RJ, Musher DM. Treatment of Clostridium difficile-associated disease: old therapies and new strategies. Lancet Infect Dis. 2005;5:549-57.

37. Crowther GS, Chilton $\mathrm{CH}$, Todhunter SL, Nicholson S, Freeman J, Wilcox MH. Recurrence of dual-strain Clostridium difficile infection in an in vitro human gut model. J Antimicrob Chemother. 2015;70:2316-21.

38. Brüggemann H, Brzuszkiewicz E, Chapeton-Montes D, Plourde L, Speck D. Genomics of Clostridium tetani. Res Microbiol. 2015;166:326-31.

39. Carman RJ, Genheimer CW, Rafii F, Park M, Hiltonsmith MF, Lyerly DM. Diversity of moxifloxacin resistance during a nosocomial outbreak of a predominantly ribotype ARU 027 Clostridium difficile diarrhea. Anaerobe. 2009;15:244-8.

40. Drudy D, Kyne L, O'Mahony R, Fanning S. gyrA mutations fluoroquinoloneresistant Clostridium difficile PCR-027 [3]. Emerg Infect Dis. 2007;13:504-5

41. Walkty A, Boyd DA, Gravel D, Hutchinson J, McGeer A, Moore D, et al. Molecular characterization of moxifloxacin resistance from Canadian Clostridium difficile clinical isolates. Diagn Microbiol Infect Dis. Elsevier B.V. 2010;66:419-24.

42. Hargreaves KR, Otieno JR, Thanki A, Blades MJ, Millard AD, Browne HP, et al. As clear as mud? Determining the diversity and prevalence of prophages in the draft genomes of estuarine isolates of Clostridium difficile. Genome Biol Evol. 2015;7:1842-55.

43. Wiedenbeck J, Cohan FM. Origins of bacterial diversity through horizontal genetic transfer and adaptation to new ecological niches. FEMS Microbiol Rev. 2011;35:957-76.

44. Castillo-Ramírez S, Harris SR, Holden MTG, He M, Parkhill J, Bentley SD, et al. The impact of recombination on $\mathrm{dN} / \mathrm{dS}$ within recently emerged bacterial clones. PLoS Pathog. 2011;7:e1002129.

45. Johnson RC. Site-specific DNA inversion by serine Recombinases. 2014;1-36.

46. Anjuwon-Foster BR, Tamayo R, Browne H, Leung W, Frank J, Schmitz A. A genetic switch controls the production of flagella and toxins in Clostridium difficile. PLoS Genet. Kearns DB, editor. John Wiley \& Sons, Inc. 2017;13: e1006701.

47. Emerson JE, Reynolds CB, Fagan RP, Shaw HA, Goulding D, Fairweather NF. A novel genetic switch controls phase variable expression of CwpV, a Clostridium difficile cell wall protein. Mol Microbiol. 2009;74:541-56.

48. Tasteyre A, Karjalainen T, Avesani V, Delmée M, Collignon A, Bourlioux P, et al. Phenotypic and genotypic diversity of the flagellin gene (fliC) among Clostridium difficile isolates from different serogroups. J Clin Microbiol. 2000; 38:3179-86.

49. Stabler RA, He M, Dawson L, Martin M, Valiente E, Corton C, et al. Comparative genome and phenotypic analysis of Clostridium difficile 027 strains provides insight into the evolution of a hypervirulent bacterium. Genome Biol. 2009:10:R102.

50. Stevenson E, Minton NP, Kuehne SA. The role of flagella in Clostridium difficile pathogenicity. Trends Microbiol. Elsevier Ltd. 2015:23:275-82.

51. Hargreaves KR, Thanki AM, Jose BR, Oggioni MR, MR C. Use of single molecule sequencing for comparative genomics of an environmental and a clinical isolate of Clostridium difficile ribotype 078. BMC Genomics. 2016;17:1020. 2.

\section{Submit your next manuscript to BioMed Central and we will help you at every step:}

- We accept pre-submission inquiries

- Our selector tool helps you to find the most relevant journal

- We provide round the clock customer support

- Convenient online submission

- Thorough peer review

- Inclusion in PubMed and all major indexing services

- Maximum visibility for your research

Submit your manuscript at www.biomedcentral.com/submit
C) Biomed Central 